\title{
Measuring network resilience through connection patterns
}

\author{
Roy Cerqueti $^{1} \quad$ Giovanna Ferraro ${ }^{2} \quad$ Antonio Iovanella ${ }^{2 *}$ \\ ${ }^{1}$ Department of Economics and Law \\ University of Macerata \\ Via Crescimbeni, 20 - 62100 Macerata, Italy \\ roy.cerqueti@unimc.it \\ ${ }^{2}$ Department of Enterprise Engineering \\ University of Rome Tor Vergata \\ Via del Politecnico, 1 - 00133 Rome, Italy. \\ giovanna.ferraro@uniroma2.it \\ antonio.iovanella@uniroma2.it
}

\begin{abstract}
Networks are at the core of modeling many engineering contexts, mainly in the case of infrastructures and communication systems. The resilience of a network, which is the property of the system capable of absorbing external shocks, is then of paramount relevance in the applications. This paper deals with this topic by advancing a theoretical proposal for measuring the resilience of a network. The proposal is based on the study of the shocks propagation along the patterns of connections among nodes. The theoretical model is tested on the real-world instances of two important airport systems in the US air traffic network: Illinois (including the hub of Chicago) and New York states (with JFK airport).
\end{abstract}

Keywords: networks; resilience; paths; weighted arcs; air traffic systems.

\section{Introduction}

Networks are an important tool used to describe and analyze the structure and dynamical behaviors of several complex systems found in the real world. In particular, network

${ }^{*}$ Corresponding author. 
representation applies to transportation systems such as airline routes [12] and railroads [29]; large communication infrastructures such as the World-Wide-Web [5] and the Internet [15]; biological systems [4] such as gene and protein interaction networks [28] and many social interaction structures [9], [16].

In the framework of networks, the interacting elements are depicted as nodes in the system and their interactions are represented as links connecting the nodes. A relevant issue relies to the behavior of networks with respect to the occurrence of external shocks.

In this respect, it is possible to distinguish the network's robustness, i.e. the capacity of the network to perform its basic functions even in the event of missing elements (nodes or links) or, to phrase this concept differently, the ability of the system to tolerate strains and to maintain its functionality in some way; and the network's resilience, i.e. the ability of a system to return to its normal state after a disturbance due to internal or external shocks by altering its processes while continuing to perform [6], [17], [23].

Literature on networks resilience is rather wide. At its core, shocks are interpreted as the removal of nodes on the basis of several different criteria (see for example: [3], [10], [11], [17]).

However, engineering applications reveal that shocks can not be associated forcefully to the disappearance of nodes. One might experience the occurrence of a shock as a perturbations whose size proxies the shock's relevance. An example can be the loss of capacity of an electricity plant due to adverse weather conditions and the corresponding damage of the entire energy system.

The network resilience is considered crucial for the different practical approaches [20], [30], [35] whereby the criticalities related to the eventual failure of nodes and links and by means of overall system tolerance [19]. Disruptive events, whether a natural disaster, intentional attacks or common failures, can have relevant impacts when they lead to the failure of network elements. Indeed, network performances are directly related to their resilience and thus to the abilities of networks in tolerating loss of important elements such as bridges or hubs [11], [21], [31].

Resilience has been studied across several different network structures and there is now knowledge regarding how specific types of networks react to specific kinds of losses 
[8]. Studies about this topic have considered a large variety of structural failures, both induced by attack or naturally occurring, which involve nodes chosen by their central position in the system, as well as those that are random.

However, the current literature on how to measure resilience is very limited [7]; [22]; [34] with most contributions focusing on the remaining part of the network after the damage. For example, some authors measured resilience through the number of node failures and link failures that can occur for a system that is still functioning [26]; others proposed an approach to evaluate the logistics of network resilience based on the redundant resource and reliability of nodes and links [33]; Ash and Newth [1] used an algorithm to evolve the complex systems that are resilient to cascading failure; Wang et al., [32] proposed a measure based on the recovery ability of a system; Ramirez-Marquez et al., [25] evaluated the resilience of community structures in networks; Miller-Hooks et al. [24] measured the maximum resilience of freight transportation network.

In this context, it is therefore relevant to analyze how an external shock that affects a node propagates along the network paths.

The aim of this paper is to introduce a new analytical measure of resilience for weighted networks that can be used when external shocks occur. The idea is to subject the network to something akin to a load test and check how it responds.

Thus, we consider the resilience of a network as its ability to absorb a shock. We introduce the concept of shock and the modality of its propagation, which is assumed to depend strongly on the weights of the arcs. The presence of weights along the links allows us to take into account the relevance given to the length of the paths between the nodes.

The intuitive basic idea is that a shock occurs at a single node and it propagates from there over all the arcs, starting from the involved node. Once the shock reaches the adjacents, it then proceeds to the adjacents of the adjacents, and so on. There is a required condition for the propagation, based on the weights on the arcs. Substantially, the shock propagates only if the propagating arcs have sufficient weight. Moreover, we also assume that an amplifying/damping effect on the shock is in force on the basis of the distance from the originating node. Details will be provided below.

We test the proposed measure of resilience on two empirical networks extracted from 
the network of US commercial airports: the Illinois state network and the New York state network. In particular, we offer the comparison between the case of unweighted airports networks - whose arcs capture the existence of a flight connection between two airports - and the weighted ones - where the weights count the number of existing daily flights connecting two airports. A discussion of the resilience of such real-world instances is carried out, along with the similarities and discrepancies between the weighted and unweighted cases.

The paper is organized as follows. Section 2 provides preliminaries and notations. Section 3 focuses on the conceptualization of the proposed measure of the resilience. Section 4 shows the analysis of the resilience on two empirical networks and offers also a discussion of the resilience in both cases of weighted and unweighted arcs. Section 5 outlines some concluding remarks.

\section{Preliminaries and notations}

A network is an analytical-geometrical structure which is able to describe a set of interconnected elements in a unified system. The basis of a network is a graph $G=(V, E)$, where $V$ is the set of $n$ nodes (or vertices) and $E$ is the set of $m$ links (or arcs). The links formalize the connections among the nodes.

Two generic nodes will be denoted by two integers $i, j=1, \ldots, n$. The presence of a link between the nodes $i$ and $j$ will be captured through a binary variable $a_{i j}$ which is 1 or 0 if the link does exist or not, respectively.

In so doing, we can fully identify $E$ through the adjacency matrix $\mathbf{A}=\left(a_{i j}\right)_{i, j=1, \ldots, n}$. The proposed setting is grounded on networks which are weighted and oriented. Specifically, two possible arcs connect $i$ and $j$ : the one from $i$ to $j$ and the one from $j$ to $i$. Accordingly, we will hereafter denote the arc from $i$ to $j$ by $i \rightarrow j$. Moreover, arcs are weighted by positive numbers which proxy the level of interaction among the nodes. We denote the weight of the arc $i \rightarrow j$ by $w_{i j}$. In general, $w_{i j} \neq w_{j i}$. By assuming that

$a_{i j}=0$ if and only if $w_{i j}=0$, we can replace the matrix $\mathbf{A}$ with the more informative weighted adjacency matrix $\mathbf{W}=\left(w_{i j}\right)_{i, j=1, \ldots, n}$. Indeed, by employing such a matrix, one can simultaneously identify the existing links and their corresponding weights. 
We point out that the weighted adjacency matrix generalizes the unweighted one, by taking $w_{i j}=1$ when the undirected arc connecting $i$ and $j$ exists.

The network is then $N=(V, \mathbf{W})$.

Two arcs are said to be consecutive if they share exactly one of the two nodes compounding them. Two consecutive arcs form a path of length 2 or, simply, a 2-path. A generic 2-path with starting node $i_{0} \in V$ can be written as $p_{i_{0}}^{(2)}=\left\{i_{0} \rightarrow i_{1} \rightarrow i_{2}\right\}$, with $i_{0}, i_{1}, i_{2}$ being three distinct nodes in $V$ and $i_{0} \rightarrow i_{1}, i_{1} \rightarrow i_{2} \in E$.

Given an integer $k \geq 3$, we can extend such a definition to the case of $k$-paths in a natural way. A generic $k$-path with starting node $i_{0} \in V$ is $p_{i_{0}}^{(k)}=\left\{i_{0} \rightarrow i_{1} \rightarrow \cdots \rightarrow\right.$ $\left.i_{k-1} \rightarrow i_{k}\right\}$, where $i_{0}, i_{1}, \ldots, i_{k-1}, i_{k}$ are distinct nodes in $V$ and $i_{h-1} \rightarrow i_{h} \in E$, for each $h=1, \ldots, k$.

Under this definition, a 1-path is simply an arc in $E$.

We collect all the $k$-paths of $N$ with starting node $i_{0}$ in a set $\mathcal{P}^{(k)}\left(i_{0}\right)$. All the $k$-paths of the network will be collected in

$$
\mathcal{P}^{(k)}=\bigcup_{i_{0} \in V} \mathcal{P}^{(k)}\left(i_{0}\right)
$$

The weight of a $k$-path can be defined by the vector of the weights of the arcs composing it. We denote such a weight by $w\left(p_{i_{0}}^{(k)}\right)=\left(w_{i_{0} i_{1}}, w_{i_{1} i_{2}}, \ldots, w_{i_{k-1} i_{k}}\right)$. A suitable aggregation of the components of the weight of $p_{i_{0}}^{(k)}$ will be discussed in the next section, in the context of the propagation of the shocks in the network.

We also denote by $\bar{k}$ the length of the longest $k$-path of the network.

\section{The measure of the resilience}

This section is devoted to the conceptualization of the measure of the resilience of the network.

The resilience of a network describes its ability to absorb an external solicitation, or shock. Such an ability is strongly dependent on the topology of the network: indeed, a solicitation of the network may appear in one of its components (a node, in general) and from the starting point it propagates through the arcs and the nodes of the network. In this context, a crucial role is played by the analysis of the characteristics of the external 
solicitation and by the features of the dynamics within the system modeled through the network. Therefore, in order to provide a definition of resilience, one needs to introduce the concept of shock and also the way in which it propagates in the network.

In our setting, a shock is an external solicitation of one of the nodes of the network. Such a local occurrence is able to destabilize the entire network by propagating over the other nodes of the network. The destabilization of the network is strongly dependent on how shocks propagate and, of course, on the entity of the shock. Indeed, shocks are not identical, and they can be of different size. We measure the size of a shock by a term $\xi \in[0,+\infty)$, with the conventional agreement that the higher the value of $\xi$, the more severe the occurred shock. The case $\xi=0$ is included for the sake of completeness, and it means no shock.

In this framework, we assume that the propagation of a shock occurring at a given node $i_{0}$ follows the route traced by the $k$-paths with starting node $i_{0}$, and depends on the values of the weights of the arcs of the $k$-path and on the distance of the nodes of the $k$-path from the shocked node. Specifically, we reasonably claim that the shock propagates if it is large enough. Moreover, we also assume that the propagation motion modifies the size of the shock. Such a modification is grounded on the fact that the passage over an arc with high (low) weight is associated to a large (small) modified size for the shock. As a further requirement, we assume that the size is affected by time, so that a discount factor acts in the propagation of the shock.

We enter the details.

Consider an integer $k$ and a $k$-path having, as a starting node, a node with a shock, namely $p_{i_{0}}^{(k)}=\left\{i_{0} \rightarrow i_{1} \rightarrow \cdots \rightarrow i_{k-1} \rightarrow i_{k}\right\} \in \mathcal{P}^{(k)}\left(i_{0}\right)$. Hypothetically, the shock propagates on a sequential basis: from $i_{0}$ to $i_{1}$, from $i_{1}$ to $i_{2}$ and so on, till the last node $i_{k}$. In real practice, the shock propagation is dampened or amplified - it depends on the specific context - as the distance from the starting node grows. As we will see, such a damping/amplifying effect might eradicate/exacerbate the action of the shock at nodes that are far enough from $i_{0}$. The reasoning behind this effect on the size of the distance from the shocked node lies in the evidence that the propagation flows tend to accumulate or lose their power as they become far from the original source. The specification of 
accumulation or lose depends on the peculiar reference context.

Furthermore, the way the shock propagates is assumed to be captured by the weight $w\left(p_{i_{0}}^{(k)}\right)$. This is a natural requirement, in that the propagation of a shock from a node to another one has necessarily to be related to the entity of the interaction between them.

According to these arguments, we introduce a discount factor $\delta \in[0,+\infty)$, which describes the damping/amplifying effect in the propagation motion of a shock with size $\xi$ on $p_{i_{0}}^{(k)}$ in a discrete time framework, as follows:

- at time $t=0$, the shock with size $\xi_{0}=\xi$ occurs at $i_{0} \in V$;

- at a generic time $t=h$, with $1 \leq h \leq k$, the shock propagates to $i_{h}$ with size $\xi_{h}$, with

$$
\xi_{h}=\left[\xi_{h-1}+w_{i_{h-1} i_{h}}\right] \delta=\xi \sum_{s=1}^{h} w_{i_{s-1} i_{s}} \delta^{h-s+1} .
$$

As already mentioned above, we include the possibility of having a stop of the propagation of the shock in our setting. Such an additional ingredient of the model meets the evidence that the weakness of the size of the shock or of the weights of the arcs might lead to the interruption of the propagation. Thus, if $h<k$, then the shock propagates to node $i_{h+1}$ only if a propagation condition is satisfied (see below). Once the shock ceases to propagate, the propagation motion stops.

Notice that the parameter $\delta$ plays the role of discount factor in the propagation motion, and formalize the action of the time in the modification of the size of the shock. In particular, $\delta \in(0,1)$ is associated to a damping of the distance from the shocked node, while $\delta>1$ captures an amplifying effect. The case $\delta=0$ is trivial, and means "no propagation", while $\delta=1$ means that the distance from the original node has no impact on the propagation of the shock.

Moreover, the definition of the propagation motion of a shock can be adapted also to the case of undirected unweighted network, bearing in mind that the unweighted network is a weighted one with unitary weights.

It is important to stress that the definition of the sizes of the propagated shock in $(2)$ is formalized by implicitly assuming that the shocks $\xi$ 's and the weights of the arcs w's share the same unit of measure. Indeed, the modified size of the shock let intervene the 
combination of the size itself at previous steps and of the weights on the arcs, which have to be then consistent under a dimensional point of view.

We are now ready to formalize the propagation condition - $P C$, hereafter - which is the requirement to be satisfied in order to avoid the stopping of the propagation motion. As already preannounced above, we assume that the shock propagates only if its size is large enough. By recalling that the longest $k$-paths in the network are the ones with $k=\bar{k}$, we introduce a vector of positive scalars $\Gamma=\left(\gamma_{1}, \ldots \gamma_{\bar{k}}\right) \in \mathbb{R}^{\bar{k}}$.

Given $h=1,2, \ldots, k$, we assume that the shock does not propagate to $i_{h}$ if and only if there exists $s=0,1,2, \ldots, h-1$ such that $\xi_{s}<\gamma_{s}$. Such a condition is evidently tailored on the individual $k$-path with initial point $i_{0}$, so that the propagation motion can proceed over some $k$-paths and stop over other ones. Such a remark suggests that the $k$-paths can be clustered on the basis of their property of satisfying the PC, and the clustering depends on the selected $\Gamma$ and on the size of the shock $\xi$. Therefore, given $\Gamma \in \mathbb{R}^{\bar{k}}$ and $\xi \in[0,+\infty)$, if the shock is propagated till the last node $i_{k}$, then we say that $p_{i_{0}}^{(k)}$ satisfies $(\Gamma, \xi)-P C$ or, simply, $p_{i_{0}}^{(k)}$ is $(\Gamma, \xi)-P C$. Vector $\Gamma$ is the $P C$-vector of the network $N$.

Clearly, specific values of $\Gamma$ and $\xi$ determine the set of the $k$-paths which are $(\Gamma, \xi)-P C$. In this respect, fixed $\Gamma \in \mathbb{R}^{k}$ and $\xi \in[0,+\infty)$, we define

$$
\mathcal{P}_{\Gamma, \xi}^{(k)}=\bigcup_{i_{0} \in V}\left\{p_{i_{0}}^{(k)} \in \mathcal{P}^{(k)}\left(i_{0}\right): p_{i_{0}}^{(k)} \text { is }(\Gamma, \xi)-P C\right\}
$$

Notice that the thresholds $\gamma$ 's are given a-priori, do not depend on the specific starting node $i_{0}$ and can be arbitrarily fixed. In principle, they can also be taken as endogenous. Indeed, one can identify the PC of the entire network by defining the minimal values of the $\gamma$ 's serving for the propagation condition over all the $k$-paths of the network. We denote such critical threshold by $\gamma^{\star}$ 's and collect them in $\Gamma^{\star}=\left(\gamma_{1}^{\star}, \ldots \gamma_{\bar{k}}^{\star}\right)$. The vector $\Gamma^{\star}$ could be defined as the critical PC-vector of the network $N$.

In general, given $\Gamma$ and $\xi$, we can define the $\Gamma-\xi$-resilience of the network as the ability of the network to stop the propagation of the shocks when the PC-vector is $\Gamma$ and the shocks have initial size $\xi_{0}=\xi$. Such an ability can be measured by taking into account the relative quantity given by the number of the $k$-paths which satisfy the PC over the total amount of $k$-paths. Furthermore, all the possible lengths $k=1, \ldots, \bar{k}$ have to be included in the definition of the measure. Therefore, the sets defined in (1) and (3) as $k$ 
varies can be efficiently employed to provide a relative measure of the resilience of $N$, once $\Gamma$ and $\xi$ are fixed.

According to this argument, we define the measure of the $\Gamma-\xi$-resilience of $N-$ and denote it by $\mu_{(\Gamma, \xi)}(N)$ - as a weighted mean of the relative number of the $k$-paths which are $(\Gamma, \xi)-P C$ as $k$ varies:

$$
\mu_{(\Gamma, \xi)}(N)=\sum_{k=1}^{\bar{k}} \theta_{k} \frac{\left|\mathcal{P}_{\Gamma, \xi}^{(k)}\right|}{\left|\mathcal{P}^{(k)}\right|},
$$

where the weights are collected in a vector of nonnegative scalars $\Theta=\left(\theta_{1}, \ldots, \theta_{\bar{k}}\right)$ such

that $\sum_{k=1}^{\bar{k}} \theta_{k}=1$. By construction, $\mu_{(\Gamma, \xi)}(N) \in[0,1]$, for each network $N, \Gamma \in \mathbb{R}^{\bar{k}}$ and $\xi \in[0,+\infty)$. Such a property allows one to compare different networks in terms of their abilities to absorb shocks. The corner cases of $\mu_{(\Gamma, \xi)}(N)=0$ and $\mu_{(\Gamma, \xi)}(N)=1$ stand for the minimum and maximum level of $\Gamma-\xi$-resilience, respectively. Indeed, in the former case the shocks with size $\xi$ are immediately absorbed; in the latter case, any shock of size $\xi$ propagates over all the available paths.

Furthermore, the presence of the weights $\theta$ 's allows one to provide different conceptualizations of $\Gamma-\xi$-resilient networks, on the basis of the relevance given to the length of the paths in the overall computation of the measure of the resilience. To distinguish the weights $\theta$ 's and the weights of the network w's, we will refer to $\theta$ as resilience weights, hereafter.

Intuitively, $\mu_{(\Gamma, \xi)}(N)$ decreases with respect to all the components of $\Gamma$ and increases with respect to $\xi$, because the $\mathrm{PC}$ becomes more restrictive if the thresholds $\gamma$ 's are large or if the size of the shock is small.

For a review of the employed notation, please refer to Table 1.

\section{Applications}

Herein we considered the analysis of the resilience on two empirical networks extracted from the network of US commercial airports [13]. The original US commercial airports network has $n=500$ nodes denoting airports and $m=2980$ directed arcs representing flight connections. We consider both the unweighted and weighted case. In the former case, we aim at exploring the resilience of the simple networks modeling the existing flight connections among airports; in the latter case, we include also the number of daily flights 


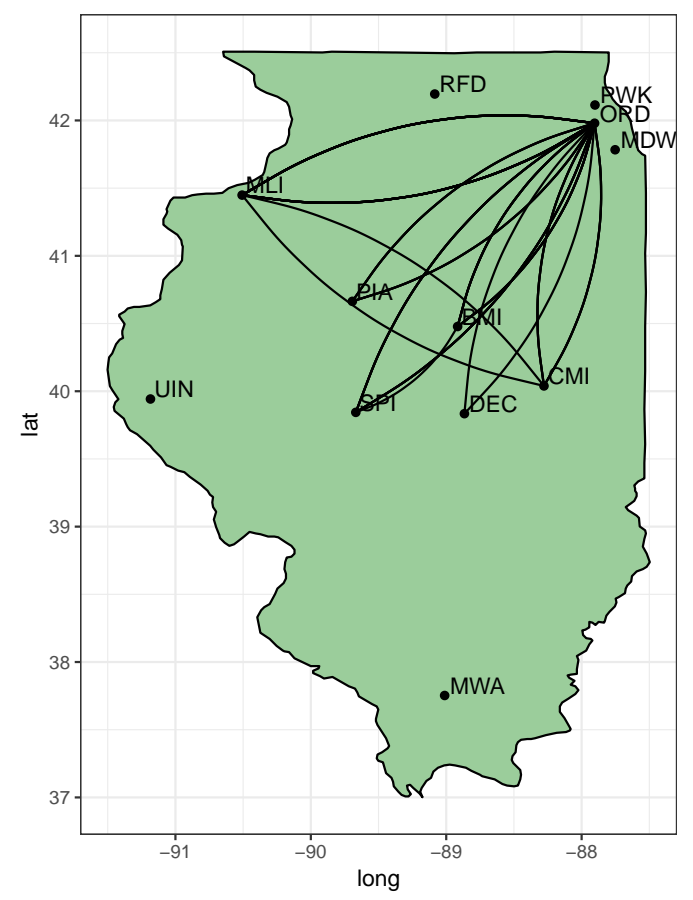

Figure 1: Map of airports and relative network routes for Illinois state, with 12 nodes and 51 arcs.

available on such connections in 2010 as weights. The weighted network has both a smallworld and scale-free organization [2]. In particular, we present the cases of the airports in Illinois (IL) and New York (NY) states. The selected networks are relatively small. This allows us to overcome the computational complexity problems, since the computation of all the simple paths grows exponentially with the size of the network [18]. However, the case studies regarding the airports in Illinois and New York states can be considered of interest being that the two airport hubs of Chicago (ORD according to International Air Transport Association - IATA - code nome) and New York (JFK) are extremely crucial in US air traffic network.

The subnetwork extracted for IL is composed of 12 nodes and 51 arcs with $\bar{k}=4$ while the NY subnetwork is composed of 21 nodes and 89 connection $\operatorname{arcs}$ with $\bar{k}=8$. Figures 1 and 2 show, respectively, the visualizations of relative networks overlapped on the maps of NY and IL states. 


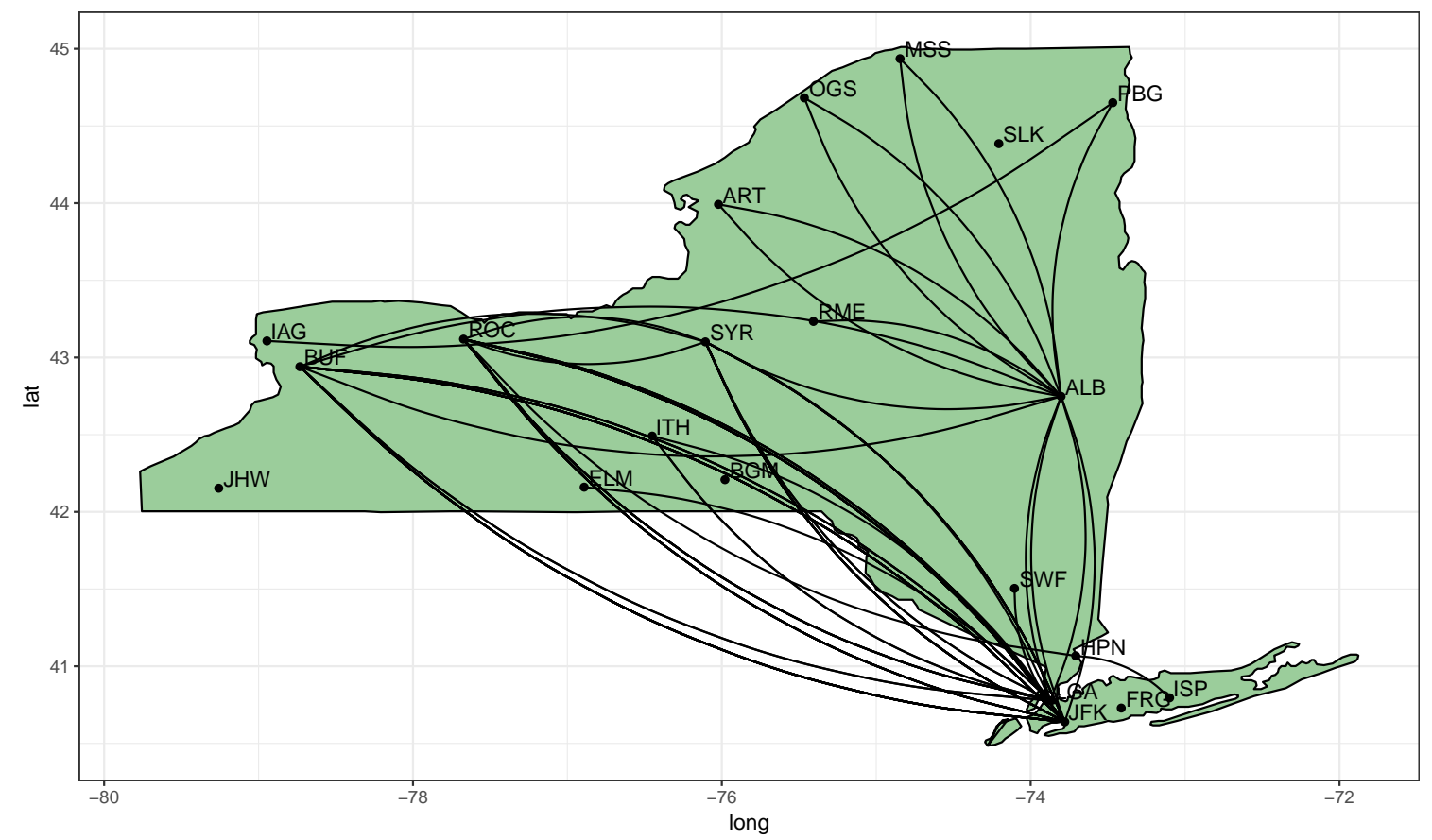

Figure 2: Map of airports and relative network routes for New York state, with 21 nodes and 89 arcs.

\subsection{Implementation details}

Formulas (3) and (4) were implemented in form of an algorithm which takes as input the network $N$, the values for $\xi$ and $\delta$ and some different settings for $\Gamma$ and $\Theta$ as described later.

For the computation of the $\mu_{(\Gamma, \xi)}(N)$, we considered $\xi=\{0,1,2, \ldots, 10\}$ and $\delta=$ $\{0,0.1,0.2, \ldots, 1\}$, thus we performed 121 computations for each network. The higher values of $\xi$ were chosen in order to be greater than $\bar{k}$.

Moreover, as already preannounced and in order to give a more detailed analysis, we performed simulations on unweighted as well as weighted networks. The analysis of the unweighted networks - which correspond to unitary weights on each arc - led to the assessment of the net response of the network to shocks under a topological point of view. The weighted case provided information on the resilience of the networks when flight connections include also the entity of their air traffic. In the considered case, weights are integers counting the number of daily flights in 2010 on each arc.

The data processing, the network analysis and all simulations are conducted using the 
software R [27] with the igraph package [14]. The datasets were obtained from the R packege tnet authored by Tore Opsahl ${ }^{1}$. Code in the $\mathrm{R}$ programming language is available on Github .

The algorithm starts computing some basic network measures, than it computes all the simple paths that origin from each node $i_{0}$ in the network via a built-in function of $\mathrm{R}$ and stores them in a list which has an item for each node and each item is the list of the paths, i.e. $\mathcal{P}^{(k)}$ as in Equation (1). The elements of such lists are then manipulated, so that all the paths are clustered on the basis of their length. After this preliminary step, the $k$-paths which are $(\Gamma, \xi)-P C$ for the considered network $N$ are marked and collected in the set $\mathcal{P}_{\Gamma, \xi}^{(k)}$, as requested by Equation (3), for each $k$.

For the identification of $\mathcal{P}_{\Gamma, \xi}^{(k)}$, three different settings for $\Gamma$ have been selected. Specifically: $\Gamma_{1}=\left\{\gamma_{i}=1, i=1, \ldots, \bar{k}\right\}$, i.e. the propagation thresholds are low and a large number of shocks propagate; more than this, all the shocks propagate when $\delta=1$; $\Gamma_{2}=\left\{\gamma_{i}=1, i=1, \ldots,\lceil\bar{k} / 2\rceil\right.$ and $\gamma_{i}=2^{i-\lceil\bar{k} / 2\rceil+1}$ for $\left.i=\lceil\bar{k} / 2\rceil+1, \ldots, \bar{k}\right\}$, i.e shocks propagation becomes more difficult as the distance from the shocked nodes increases; finally, $\Gamma_{3}=\left\{\gamma_{i}=2^{\lfloor\bar{k} / 2\rfloor-i+1}\right.$ for $i=1, . .\lfloor\bar{k} / 2\rfloor$ and $\left.\gamma_{i}=1, i=\lfloor\bar{k} / 2\rfloor+1, \ldots, \bar{k}\right\}$, i.e. the propagation is obstructed along shortest simple paths with a decreasing geometric evolution.

After the identification of $\mathcal{P}_{\Gamma, \xi}^{(k)}$, for each $k$, we are finally able to compute $\mu_{(\Gamma, \xi)}(N)$ by means of Equation (4). Regarding the resilience weights in $\Theta$, we considered as first setting $\Theta_{1}$ whose $i$-th component is $\theta_{i}=1 / \bar{k}$, for $i=1, \ldots, \bar{k}$; a second is $\Theta_{2}$ with $\theta_{i}=1 / 2^{i}$ for each $i=1, \ldots, \bar{k}-1$ and $\theta_{\bar{k}}=\theta_{\bar{k}-1}$; a third setting is $\Theta_{3}$ with $\theta_{i}=1 / 2^{\bar{k}-i+1}$ for each $i=2, \ldots, \bar{k}-1$ and $\theta_{1}=\theta_{2}$.

The first setting considers uniform values of $\theta$ 's while in the last two settings, all coefficients are taken from a geometric progression of decreasing and increasing type.

\subsection{Illinois state network}

Figures 3 and 4 report the results for the IL airports for $\Gamma_{1}$ and the three different settings of $\Theta$ for the unweighted and weighted cases, respectively.

\footnotetext{
${ }^{1}$ http: //toreopsahl.com

${ }^{2}$ https://github.com/iovanella/iovanella.github.io/tree/master/Resilience
} 
In the unweighted case and in all the considered settings, resilience is such that for small values of the $\xi$ 's and $\delta$ 's, the value of $\mu_{(\Gamma, \xi)}(N)$ is small and increases with respect to $\delta$ and $\xi$, becoming equals to 1 when $\delta$ and $\xi$ are large enough. However, some remarkable differences emerge.

For cases $\Theta_{1}$ and $\Theta_{3}$, small values of the discount factor leads to hard shock propagation, even if the size of the shock is large. This is not longer true for $\Theta_{2}$, where an enlargement of the shock is able to increase the level of the resilience also when the discount factor is at a low level. This outcome is due to the more prominent role played by the shortest paths in the first and third setting when compared to the second one, where the shortest paths are strongly penalized by the resilience weights. In this respect, one should also consider that the action of the discount is more evident as the distance with the shocked node increases.
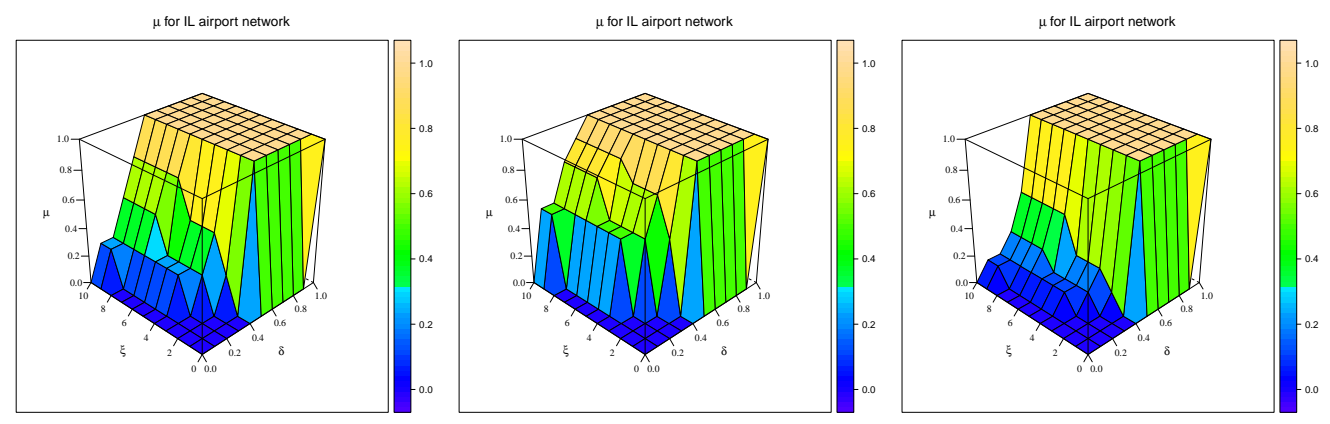

Figure 3: Measures for the $\Gamma-\xi$-resilience $\mu_{(\Gamma, \xi)}(N)$ in case of IL state airports and no weights for $\Gamma_{1}=\left\{\gamma_{i}=1, i=1, \ldots, \bar{k}\right\}$ and $\Theta_{1}=\left\{\theta_{i}=1 / \bar{k}\right.$, for $\left.i=1, \ldots, \bar{k}\right\}$ (left), $\Theta_{2}=\left\{\theta_{i}=1 / 2^{i}\right.$ for each $i=1, \ldots, \bar{k}-1$ and $\left.\theta_{\bar{k}}=\theta_{\bar{k}-1}\right\}$ (center) and $\Theta_{3}=\left\{\theta_{i}=1 / 2^{\bar{k}-i+1}\right.$ for each $i=2, \ldots, \bar{k}-1$ and $\left.\theta_{1}=\theta_{2}\right\}$ (right). Different values of $\xi$ are reported on $x$-axis, values of $\delta$ on $y$-axis and values of $\mu_{(\Gamma, \xi)}(N)$ on $z$-axis.

Similar arguments can also explain the discrepancies in the rate of growth of the resilience with respect to $\delta$. Indeed, the increase $\mu_{(\Gamma, \xi)}(N)$ is faster for $\Theta_{3}$, while the lower rate can be observed in the $\Theta_{2}$ case. Specifically, for $\Theta_{3}$ and for each value of $\xi$, we can identify the resilience as collapsing to a certain degree, with the network passing rapidly from resilience equal to 1 to very low resilience when $\delta$ moves slightly below 0.5 .

The same collapse of the resilience can be observed for all the cases when the size of the shock goes below 2. Thus, one can argue that there exists a critical threshold for the 

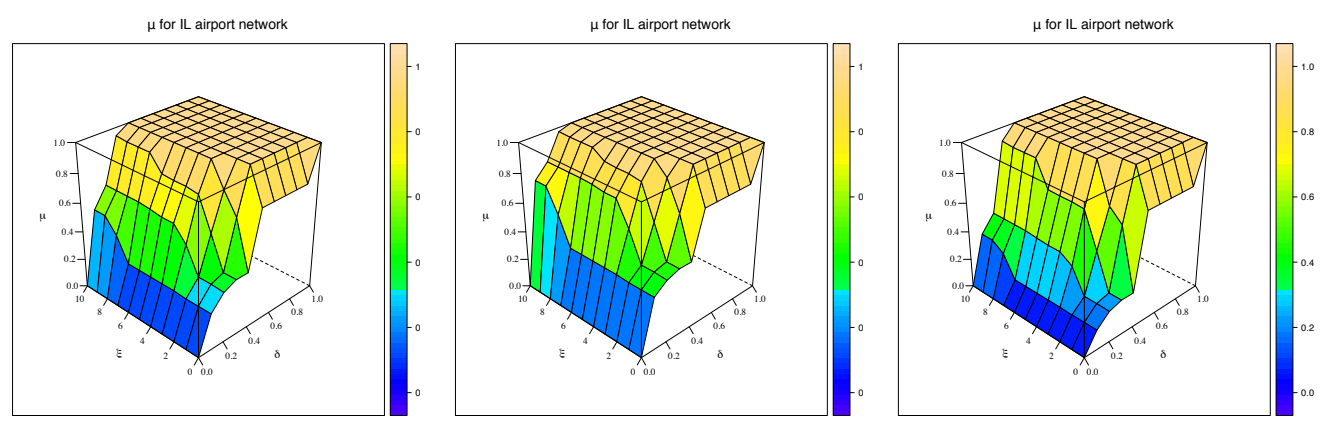

Figure 4: Measures for the $\Gamma-\xi$-resilience $\mu_{(\Gamma, \xi)}(N)$ in case of IL state airports and weights as the number of daily flights for $\Gamma_{1}=\left\{\gamma_{i}=1, i=1, \ldots, \bar{k}\right\}$ and $\Theta_{1}=\left\{\theta_{i}=1 / \bar{k}\right.$, for $i=1, \ldots, \bar{k}\}$ (left), $\Theta_{2}=\left\{\theta_{i}=1 / 2^{i}\right.$ for each $i=1, \ldots, \bar{k}-1$ and $\left.\theta_{\bar{k}}=\theta_{\bar{k}-1}\right\}$ (center) and $\Theta_{3}=\left\{\theta_{i}=1 / 2^{\bar{k}-i+1}\right.$ for each $i=2, \ldots, \bar{k}-1$ and $\left.\theta_{1}=\theta_{2}\right\}$ (right). Different values of $\xi$ are reported on $x$-axis, values of $\delta$ on $y$-axis and values of $\mu_{(\Gamma, \xi)}(N)$ on $z$-axis.

shock leading to unitary resilience, for each value of the discount factor. This is totally in agreement with $\Gamma_{1}$, which has components with minimum value. In the specific case of the IL airports, it is then associated to the propagation of all the shocks large enough when $\delta<1$ or all the shocks when $\delta=1$.

When we consider the weighted case, all the observations above remain valid but the resilience reaches values closer to one for smaller values of $\delta$. This is due to both Equation (2) as well as the propagation condition. Indeed, given $\delta$, the the propagation condition is more easily satisfied in the weighted case than in the unweighted one, and the motion of the shock becomes more difficult to be stopped.

When we consider $\Gamma_{2}$ (see Figure 5 for the unweighted case and Figure 6 for the weighted case), the $\gamma$ 's are constant for the first $\bar{k} / 2$ values, and then they start to grow; thus, we are penalizing longest simple paths. In this case (with $\Theta_{1}$ ), the effect of $\delta$ is more prominent than the one of $\xi$, and $\mu_{(\Gamma, \xi)}(N)$ tends to 1 for high values of $\delta$. Such a trend can be observed also for $\Theta_{2}$ and $\Theta_{3}$. However, $\Theta_{2}$ is much more similar to $\Theta_{1}$ than $\Theta_{3}$, because its longest paths are penalized from the $\gamma$ 's also in this setting; thus, the damping effect of the second part of $\Theta_{2}$ in the resilience weight vector seems to be hardly noticeable. The penalization of the longest path is also the reason for the absence of an evident collapse effect for $\Theta_{3}$, because shock propagation is impeded on longest paths by $\gamma$ 's and on shortest paths by $\theta$ 's. 

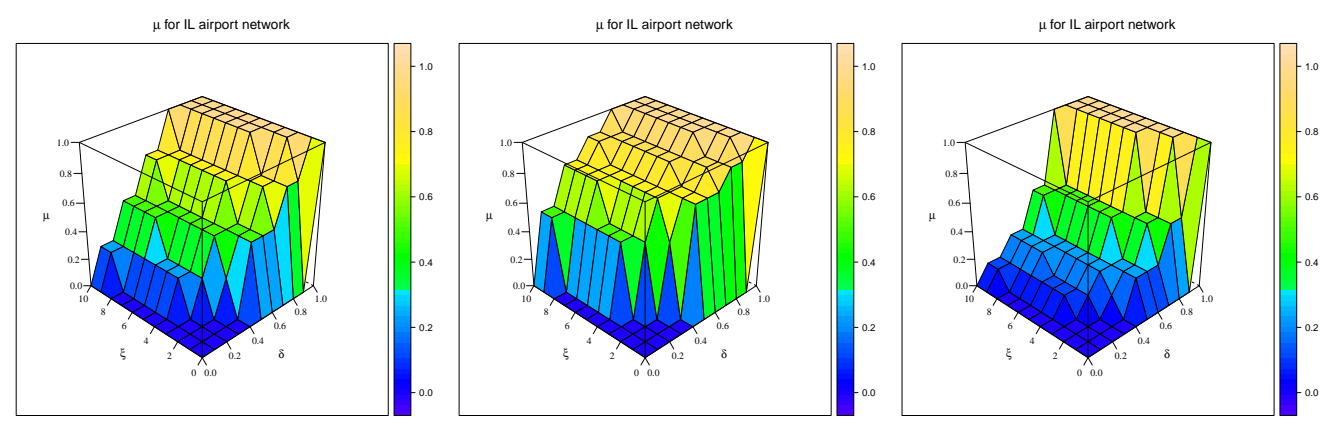

Figure 5: Measures for the $\Gamma-\xi$-resilience $\mu_{(\Gamma, \xi)}(N)$ in case of IL state airports and no weights for $\Gamma_{2}=\left\{\gamma_{i}=1, i=1, \ldots,\lceil\bar{k} / 2\rceil\right.$ and $\gamma_{i}=2^{i-\lceil\bar{k} / 2\rceil+1}$ for $\left.i=\lceil\bar{k} / 2\rceil+1, \ldots, \bar{k}\right\}$ and $\Theta_{1}=\left\{\theta_{i}=1 / \bar{k}\right.$, for $\left.i=1, \ldots, \bar{k}\right\}$ (left), $\Theta_{2}=\left\{\theta_{i}=1 / 2^{i}\right.$ for each $i=1, \ldots, \bar{k}-1$ and $\left.\theta_{\bar{k}}=\theta_{\bar{k}-1}\right\}$ (center) and $\Theta_{3}=\left\{\theta_{i}=1 / 2^{\bar{k}-i+1}\right.$ for each $i=2, \ldots, \bar{k}-1$ and $\left.\theta_{1}=\theta_{2}\right\}$ (right). Different values of $\xi$ are reported on $x$-axis, values of $\delta$ on $y$-axis and values of $\mu_{(\Gamma, \xi)}(N)$ on $z$-axis.

In the weighted case, we can observe that for $\Theta_{1}$ and $\Theta_{3}$ the concavity of the surfaces changes, showing higher marginal values for increasing $\delta$, i.e. the value of $\mu_{(\Gamma, \xi)}(N)$ approaches to one faster.
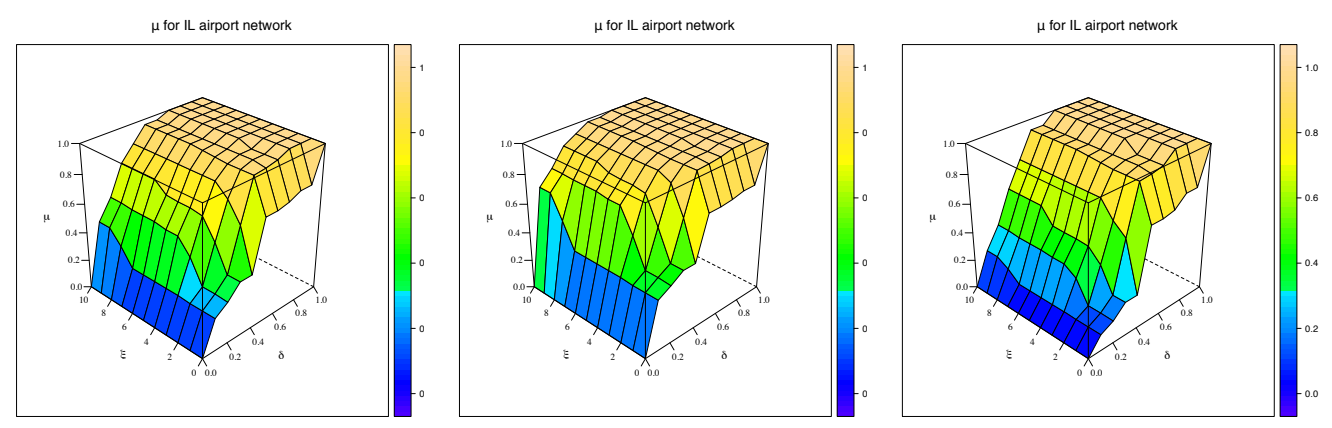

Figure 6: Measures for the $\Gamma-\xi$-resilience $\mu_{(\Gamma, \xi)}(N)$ in case of IL state airports and weights as the number of daily flights for $\Gamma_{2}=\left\{\gamma_{i}=1, i=1, \ldots,\lceil\bar{k} / 2\rceil\right.$ and $\gamma_{i}=2^{i-\lceil\bar{k} / 2\rceil+1}$ for $i=\lceil\bar{k} / 2\rceil+1, \ldots, \bar{k}\}$ and $\Theta_{1}=\left\{\theta_{i}=1 / \bar{k}\right.$, for $\left.i=1, \ldots, \bar{k}\right\}$ (left), $\Theta_{2}=\left\{\theta_{i}=1 / 2^{i}\right.$ for each $i=1, \ldots, \bar{k}-1$ and $\left.\theta_{\bar{k}}=\theta_{\bar{k}-1}\right\}$ (center) and $\Theta_{3}=\left\{\theta_{i}=1 / 2^{\bar{k}-i+1}\right.$ for each $i=2, \ldots, \bar{k}-1$ and $\left.\theta_{1}=\theta_{2}\right\}$ (right). Different values of $\xi$ are reported on $x$-axis, values of $\delta$ on $y$-axis and values of $\mu_{(\Gamma, \xi)}(N)$ on $z$-axis.

The third setting of $\Gamma$ is associated to the penalization of the shortest simple paths, so that the shock does not propagate unless it has a high value and the discount factor is high as well. Figure 7 (unweighted case) and Figure 8 (weighted case) report the results, which 

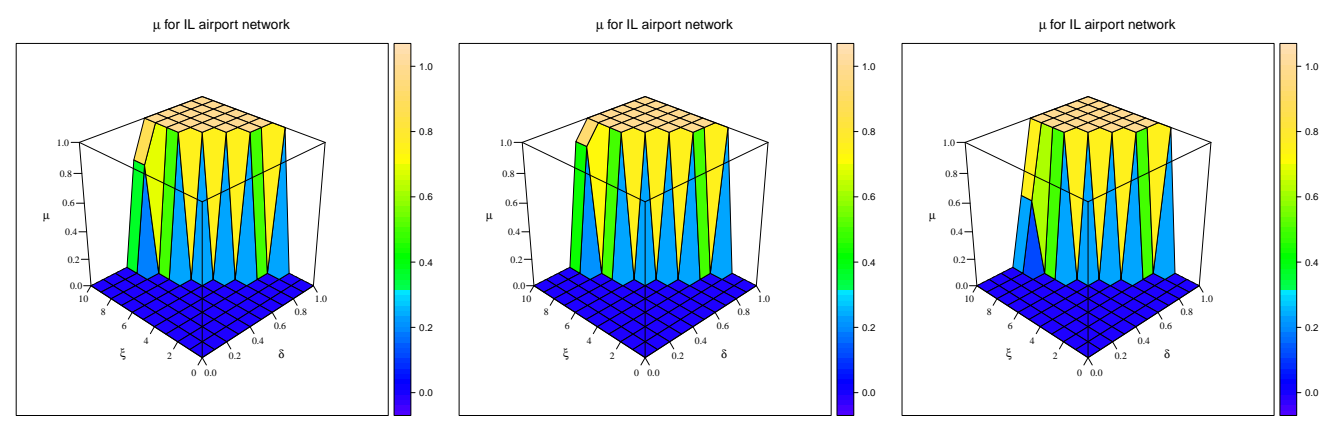

Figure 7: Measures for the $\Gamma-\xi$-resilience $\mu_{(\Gamma, \xi)}(N)$ in case of IL state airports and no weights for $\Gamma_{3}=\left\{\gamma_{i}=2^{\lfloor\bar{k} / 2\rfloor-i+1}\right.$ for $i=1, . .\lfloor\bar{k} / 2\rfloor$ and $\left.\gamma_{i}=1, i=\lfloor\bar{k} / 2\rfloor+1, \ldots, \bar{k}\right\}$ and $\Theta_{1}=\left\{\theta_{i}=1 / \bar{k}\right.$, for $\left.i=1, \ldots, \bar{k}\right\}$ (left), $\Theta_{2}=\left\{\theta_{i}=1 / 2^{i}\right.$ for each $i=1, \ldots, \bar{k}-1$ and $\left.\theta_{\bar{k}}=\theta_{\bar{k}-1}\right\}$ (center) and $\Theta_{3}=\left\{\theta_{i}=1 / 2^{\bar{k}-i+1}\right.$ for each $i=2, \ldots, \bar{k}-1$ and $\left.\theta_{1}=\theta_{2}\right\}$ (right). Different values of $\xi$ are reported on $x$-axis, values of $\delta$ on $y$-axis and values of $\mu_{(\Gamma, \xi)}(N)$ on $z$-axis.

are similar for all settings of $\Theta$ while, for the weighted case, the growth of the resilience is build through a smoother parameters surface.
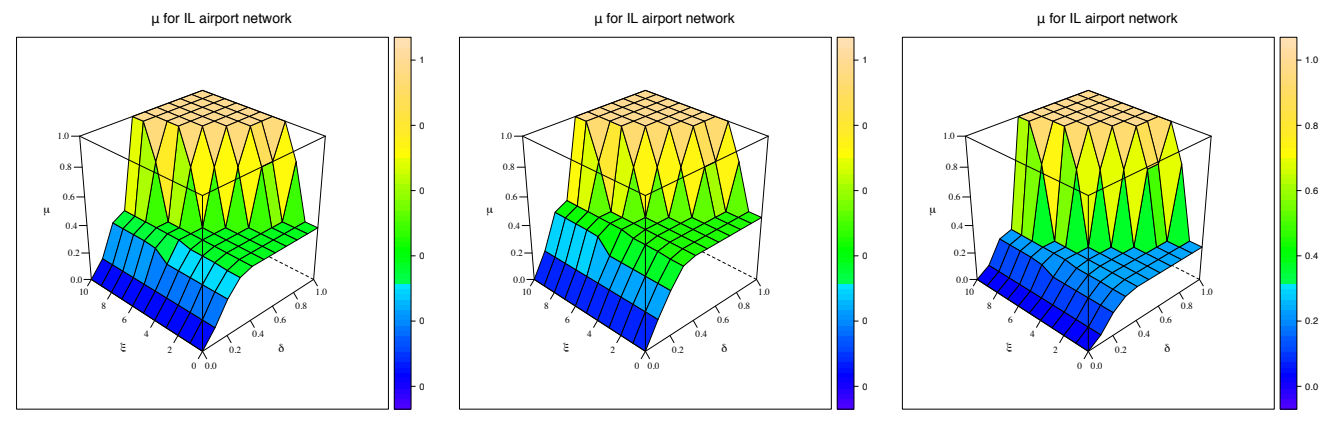

Figure 8: Measures for the $\Gamma-\xi$-resilience $\mu_{(\Gamma, \xi)}(N)$ in case of IL state airports and weights as the number of daily flights for $\Gamma_{3}=\left\{\gamma_{i}=2^{\lfloor\bar{k} / 2\rfloor-i+1}\right.$ for $i=1, . .\lfloor\bar{k} / 2\rfloor$ and $\gamma_{i}=1$, $i=\lfloor\bar{k} / 2\rfloor+1, \ldots, \bar{k}\}$ and $\Theta_{1}=\left\{\theta_{i}=1 / \bar{k}\right.$, for $\left.i=1, \ldots, \bar{k}\right\}$ (left), $\Theta_{2}=\left\{\theta_{i}=1 / 2^{i}\right.$ for each $i=1, \ldots, \bar{k}-1$ and $\left.\theta_{\bar{k}}=\theta_{\bar{k}-1}\right\}$ (center) and $\Theta_{3}=\left\{\theta_{i}=1 / 2^{\bar{k}-i+1}\right.$ for each $i=2, \ldots, \bar{k}-1$ and $\left.\theta_{1}=\theta_{2}\right\}$ (right). Different values of $\xi$ are reported on $x$-axis, values of $\delta$ on $y$-axis and values of $\mu_{(\Gamma, \xi)}(N)$ on $z$-axis.

\subsection{New York state network}

In Figures 9 (unweighted) and 10 (weighted) we report the measures of the $\Gamma_{1}-\xi$-resilience for the NY state airport network. The behavior is very similar to the one of the previous 
network in settings $\Theta_{1}$ and $\Theta_{2}$. Differently, for $\Theta_{3}$, the growth of the resilience is very clear for $\delta$ going above 0.4 in the unweighted case while, for the weighted network, the resilience profile is smoothed and has values different from zero even for small values of $\delta$.

When we set $\Gamma_{2}$ and we observe the unweighted case (see Figure 11), we note that for $\Theta_{1}$ the behavior is similar to the IL network, even if the level of the resilience of the NY network is below that of IL (compare Figure 11 with Figure 5). When we consider $\Theta_{2}$, the surface is smoothed in comparison to the same case for the IL network and it is quite

similar to the $\Gamma_{1}$ setting. For $\Theta_{3}$, the values of $\mu_{(\Gamma, \xi)}(N)$ are equal to 1 only for high values of $\xi$ and $\delta$. Indeed, the action of the $\gamma$ 's, which contribute to emphasize the relevance of the short paths in the assessment of the resilience, is more effective than that of the $\theta$ 's, which reduce the contribution of the short paths in the definition of $\mu_{(\Gamma, \xi)}(N)$.

When we move to the weighted case we can observe in Figure 12 similar behaviors of the measure of the $\Gamma-\xi$-resilience, with similar change in convexity.

The discrepancies between NY and IL networks can be explained by their dimensions, being that $\bar{k}=8$ for the former and $\bar{k}=4$ for the latter. In the same light, one should interpret also the case of $\Gamma_{3}$.

Indeed, when considering the NY airports, all the values of $\mu_{(\Gamma, \xi)}(N)$ for the unweighted case are equal to 0 and Figures are not shown. In this setting one has $\gamma_{1}>10$, which is the maximum value of the available $\xi$, so that propagation cannot occur. In the case of weighted network we obtain results different from zero (see Figure 13) since the weights impact on Equation (2) and thus, on the propagation condition.

\subsection{Results implications}

The analysis of the simulation experiments leads to some general comments. One of the main results regards the difference between the unweighted case and the weighted one. In the former, we observe the network's response to shock when we consider only the topological structure, i.e the configurations of the arcs of the network and the corresponding patterns of paths. In so doing, we are taking into account the presence of the connection flights among the airports and the consequence of a shock in this context; in the latter, we move our attention towards the inclusion of the entity of the connections through the 

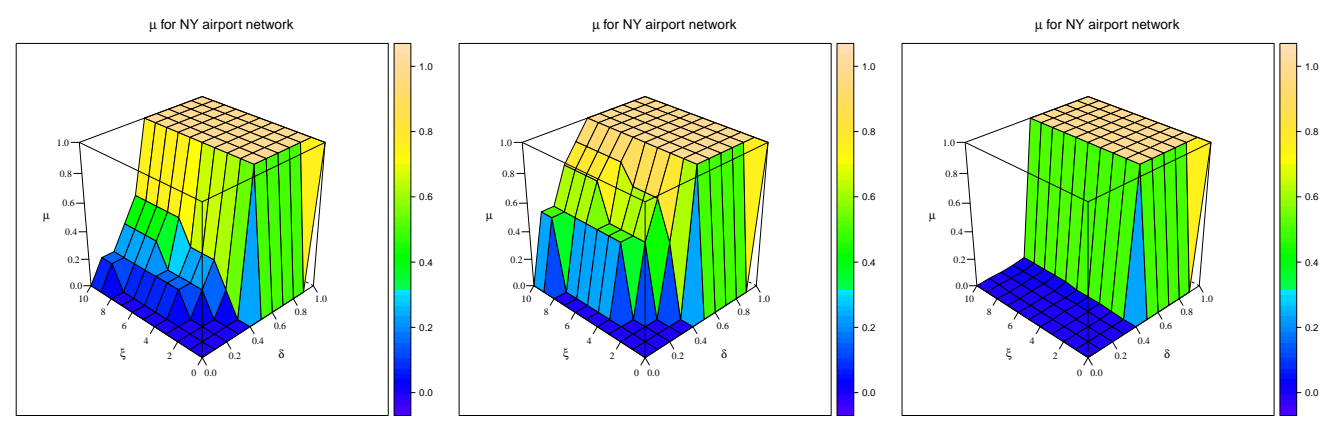

Figure 9: Measures for the $\Gamma-\xi$-resilience $\mu_{(\Gamma, \xi)}(N)$ in case of NY state airports and no weights for $\Gamma_{1}=\left\{\gamma_{i}=1, i=1, \ldots, \bar{k}\right\}$ and $\Theta_{1}=\left\{\theta_{i}=1 / \bar{k}\right.$, for $\left.i=1, \ldots, \bar{k}\right\}$ (left), $\Theta_{2}=\left\{\theta_{i}=1 / 2^{i}\right.$ for each $i=1, \ldots, \bar{k}-1$ and $\left.\theta_{\bar{k}}=\theta_{\bar{k}-1}\right\}$ (center) and $\Theta_{3}=\left\{\theta_{i}=1 / 2^{\bar{k}-i+1}\right.$ for each $i=2, \ldots, \bar{k}-1$ and $\left.\theta_{1}=\theta_{2}\right\}$ (right). Different values of $\xi$ are reported on $x$-axis, values of $\delta$ on $y$-axis and values of $\mu_{(\Gamma, \xi)}(N)$ on $z$-axis.
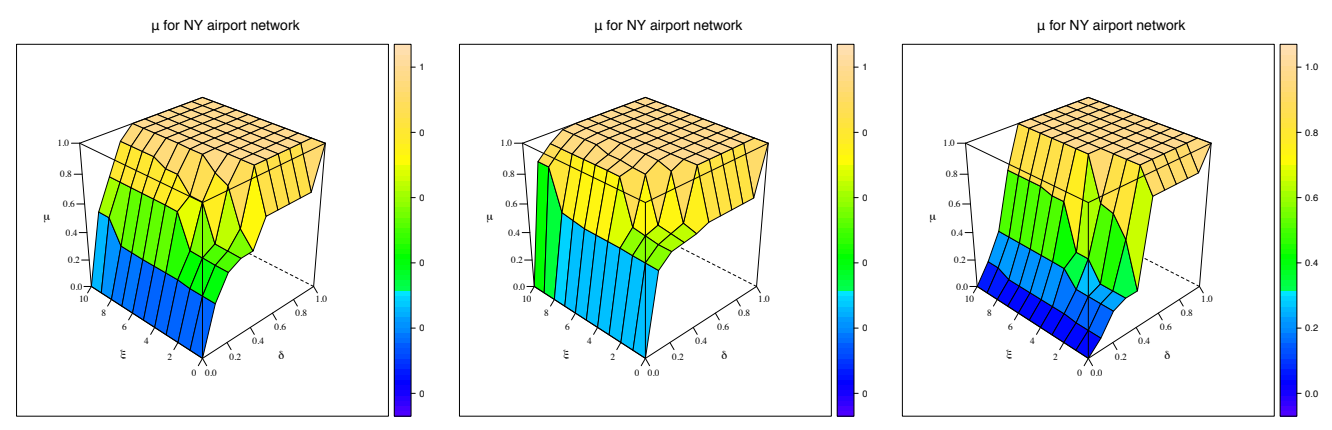

Figure 10: Measures for the $\Gamma-\xi$-resilience $\mu_{(\Gamma, \xi)}(N)$ in case of NY state airports and weights as the number of daily flights for $\Gamma_{1}=\left\{\gamma_{i}=1, i=1, \ldots, \bar{k}\right\}$ and $\Theta_{1}=\left\{\theta_{i}=1 / \bar{k}\right.$, for $i=1, \ldots, \bar{k}\}$ (left), $\Theta_{2}=\left\{\theta_{i}=1 / 2^{i}\right.$ for each $i=1, \ldots, \bar{k}-1$ and $\left.\theta_{\bar{k}}=\theta_{\bar{k}-1}\right\}$ (center) and $\Theta_{3}=\left\{\theta_{i}=1 / 2^{\bar{k}-i+1}\right.$ for each $i=2, \ldots, \bar{k}-1$ and $\left.\theta_{1}=\theta_{2}\right\}$ (right). Different values of $\xi$ are reported on $x$-axis, values of $\delta$ on $y$-axis and values of $\mu_{(\Gamma, \xi)}(N)$ on $z$-axis.

introduction of daily traffic (in the reference year 2010).

When the unweighted networks are considered, our conceptualization of resilience aims at describing the effects of the shocks in the connecting paths among the airports. In so doing, we are providing a detailed analysis of the network on the basis of the spatial location of the airports in the system and how they interact in presence of threats spreading from the nodes.

The presence of weights allows to introduce a quantification of the economic value on the network.For instance, the economic flow generated by a specific airport can be obtained 

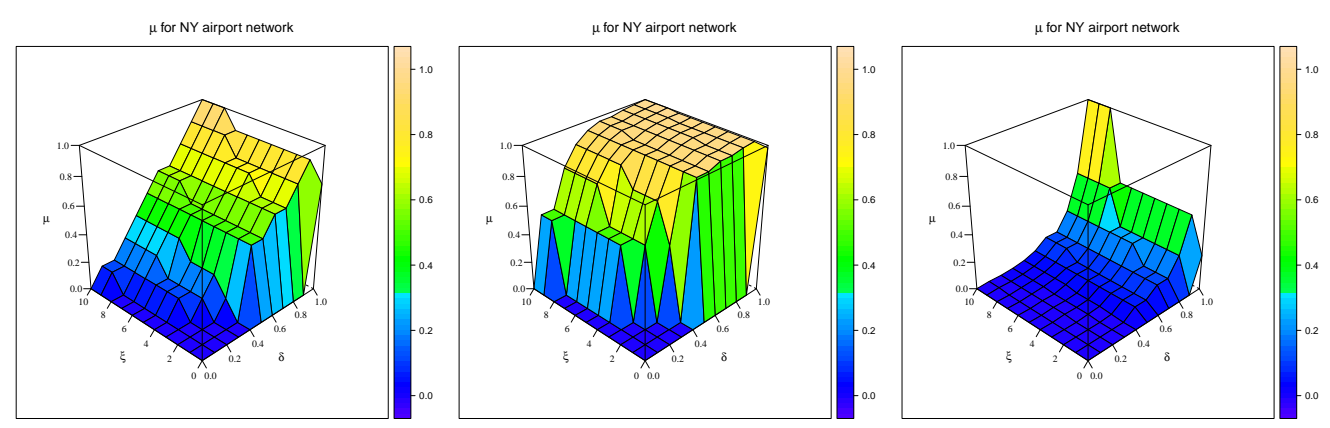

Figure 11: Measures for the $\Gamma-\xi$-resilience $\mu_{(\Gamma, \xi)}(N)$ in case of NY state airports and no weights for $\Gamma_{2}=\left\{\gamma_{i}=1, i=1, \ldots,\lceil\bar{k} / 2\rceil\right.$ and $\gamma_{i}=2^{i-\lceil\bar{k} / 2\rceil+1}$ for $\left.i=\lceil\bar{k} / 2\rceil+1, \ldots, \bar{k}\right\}$ and $\Theta_{1}=\left\{\theta_{i}=1 / \bar{k}\right.$, for $\left.i=1, \ldots, \bar{k}\right\}$ (left), $\Theta_{2}=\left\{\theta_{i}=1 / 2^{i}\right.$ for each $i=1, \ldots, \bar{k}-1$ and $\left.\theta_{\bar{k}}=\theta_{\bar{k}-1}\right\}$ (center) and $\Theta_{3}=\left\{\theta_{i}=1 / 2^{\bar{k}-i+1}\right.$ for each $i=2, \ldots, \bar{k}-1$ and $\left.\theta_{1}=\theta_{2}\right\}$ (right). Different values of $\xi$ are reported on $x$-axis, values of $\delta$ on $y$-axis and values of $\mu_{(\Gamma, \xi)}(N)$ on $z$-axis.
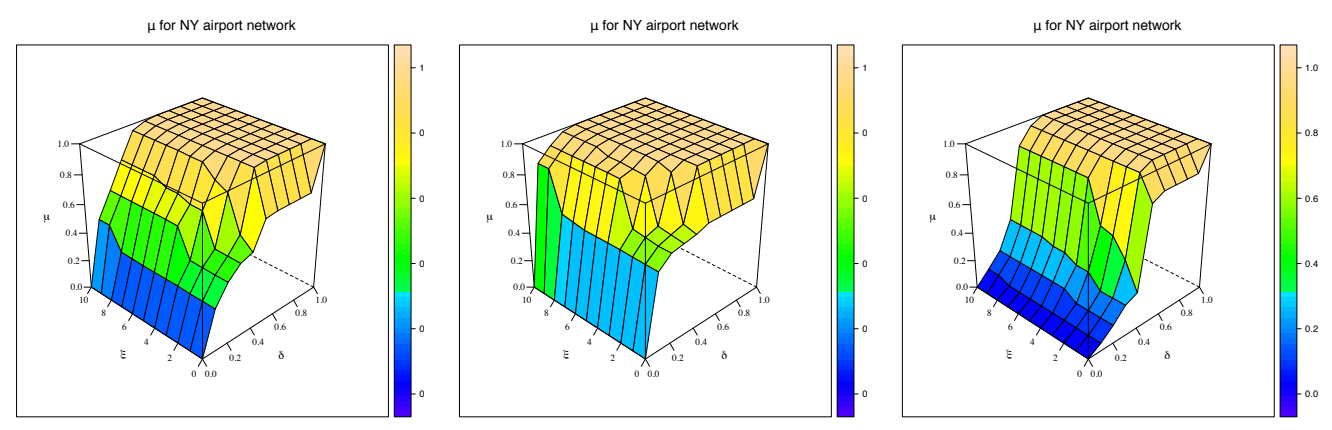

Figure 12: Measures for the $\Gamma-\xi$-resilience $\mu_{(\Gamma, \xi)}(N)$ in case of NY state airports and weights as the number of daily flights for $\Gamma_{2}=\left\{\gamma_{i}=1, i=1, \ldots,\lceil\bar{k} / 2\rceil\right.$ and $\gamma_{i}=2^{i-\lceil\bar{k} / 2\rceil+1}$ for $i=\lceil\bar{k} / 2\rceil+1, \ldots, \bar{k}\}$ and $\Theta_{1}=\left\{\theta_{i}=1 / \bar{k}\right.$, for $\left.i=1, \ldots, \bar{k}\right\}$ (left), $\Theta_{2}=\left\{\theta_{i}=1 / 2^{i}\right.$ for each $i=1, \ldots, \bar{k}-1$ and $\left.\theta_{\bar{k}}=\theta_{\bar{k}-1}\right\}$ (center) and $\Theta_{3}=\left\{\theta_{i}=1 / 2^{\bar{k}-i+1}\right.$ for each $i=2, \ldots, \bar{k}-1$ and $\left.\theta_{1}=\theta_{2}\right\}$ (right). Different values of $\xi$ are reported on $x$-axis, values of $\delta$ on $y$-axis and values of $\mu_{(\Gamma, \xi)}(N)$ on $z$-axis.

by looking also at the value of tickets sales related to the daily departures. In this context, the economic value of the network varies in presence of a sudden variation - an exogenous shock, here - of the price of the air tickets. The magnitude of the variation drives the effects on the economic value of the network, hence leading to a specific definition of the resilience. The action of the discount factor - which amplifies or dampens the propagation of the shock - is endogenously determined by the customized design of the network. 

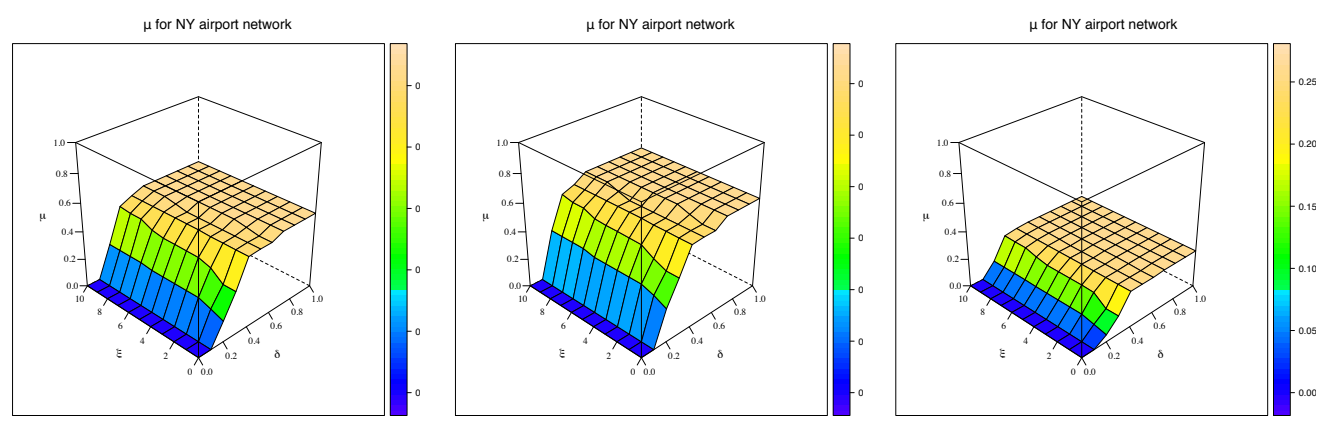

Figure 13: Measures for the $\Gamma-\xi$-resilience $\mu_{(\Gamma, \xi)}(N)$ in case of NY state airports and weights as the number of daily flights for $\Gamma_{3}=\left\{\gamma_{i}=1, i=1, \ldots,\lceil\bar{k} / 2\rceil\right.$ and $\gamma_{i}=2^{i-\lceil\bar{k} / 2\rceil+1}$ for $i=\lceil\bar{k} / 2\rceil+1, \ldots, \bar{k}\}$ and $\Theta_{1}=\left\{\theta_{i}=1 / \bar{k}\right.$, for $\left.i=1, \ldots, \bar{k}\right\}$ (left), $\Theta_{2}=\left\{\theta_{i}=1 / 2^{i}\right.$ for each $i=1, \ldots, \bar{k}-1$ and $\left.\theta_{\bar{k}}=\theta_{\bar{k}-1}\right\}$ (center) and $\Theta_{3}=\left\{\theta_{i}=1 / 2^{\bar{k}-i+1}\right.$ for each $i=2, \ldots, \bar{k}-1$ and $\left.\theta_{1}=\theta_{2}\right\}$ (right). Different values of $\xi$ are reported on $x$-axis, values of $\delta$ on $y$-axis and values of $\mu_{(\Gamma, \xi)}(N)$ on $z$-axis.

If we consider the two corner cases of resilience, i.e. $\mu_{(\Gamma, \xi)}(N)=0$ and $\mu_{(\Gamma, \xi)}(N)=1$, we can suppose that in the former case, the overall value of the network remains the same because the shock is too low or the factor $\delta$ has blocked the shock propagation, while in the second case, the overall value of the network is driven to zero meaning that the shock was strong enough or the discount factor was not able to block the propagation. All the intermediate values of $\mu_{(\Gamma, \xi)}(N)$ impact on the overall value of the network according to the surface shapes, as illustrated in Sections 4.2 and 4.3 for different settings of $\xi, \delta, \Gamma$ and $\Theta$.

A general consideration emerge, from all the settings of $\Theta$. Indeed, one can observe the effect generated by the size of the network, so that bigger networks are associated to a smaller relevance of longer paths. This evidence is explained by considering that propagation motion becomes more complicated in large networks, and shock reach hardly nodes which are far from their source. Moreover, the effect of $\delta$ is more relevant than the shock intensity $\xi$. This last consideration has an important engineering consequence, because it suggests that the design of a network which ensure a certain level of $\delta$ assures to absorb shock of relative high values. 


\section{Conclusive remarks}

The analysis of the resilience of networks and their attitude to wards absorbing external shocks is crucial for the various implications related to reliability and functionality of real-world engineering systems.

The aim of this paper is to propose a new measure of network resilience based on the study of the shocks propagation along the patterns of connections among nodes.

In our setting, shocks are assumed to have a positive size and a tendency of not necessarily removing nodes from the network. They propagate in an amplified or dampened way through a suitably defined discount factor, with such a propagation proceeding under the fulfilment of a predefined condition involving the size of the shocks and the weights of the arcs.

The resilience measure is conceptualized as a weighted combination of the cardinality of the sets collecting the paths with different lengths, whereby the resilience weights are able to assign relevance to the short or long paths in the networks.

We test the measure on two real airport systems, with a special reference to the cases of weighted and unweighted networks. The comparison between the empirical instances suggests the incidence of the size of the network on shock propagation, as well as on the resilience. Indeed, for large networks, propagation suffers from difficulty in reaching nodes that are far in respect to the others.

Results state that the resilience is strongly dependent on the resilience weights associated to the short paths, and highlight a prominent role of the discount factor in determining the ability of the network to absorb shocks. This behavior suggests that it is possible to design resilient network, with a strong ability to absorb external shocks, by imposing a propagation pattern on the basis of a certain level of the discount factor.

The proposed resilience measure might then be of usefulness to the engineers for the identification of the vulnerabilities of systems and for designing more resilient infrastructures. 


\section{References}

[1] Ash J. and Newth D. (2007). Optimizing complex network for resilience against cascading failure. Physica A: Statistical Mechanics and its Applications, 380, pp. 673-683.

[2] Barrat A., Barthélemy M., Pastor-Satorass R., Vespignani A. (2004). The architecture of complex weighted networks, Proceedings of the National Academy of Sciences of the United States of America, vol. 101, no. 11, pp 3747-3752.

[3] Albert R., Jeong H., and Barabási AL, (2000). Error and attack tolerance of complex networks Nature, vol. 406, pp. 378-382.

[4] Alon U. (2003). Biological Networks: The Tinkerer as an Engineer Science, vol. 301, pp. 1866-1867.

[5] Barabási AL, Albert R and Jeong H (2000). Scale-free characteristics of random networks: the topology of the world-wide web, Physica A: Statistical Mechanics and its Applications, vol. 281, issues 1-4, pp. 69-77.

[6] Barabási AL, Network Science, Cambridge University Press, UK, 2016.

[7] Barker K., Ramirez-Marquez J.E., Rocco C.M.(2013). Resilience-based network component importance measures. Reliability Engineering and System Safety, vol. 117, pp. 89-97.

[8] Berche B., von Ferber C., Holovatch T., and Holovatch Yu. (2009). Resilience of public transport networks against attacks The European Physical Journal B, vol. 71, issue 1, pp. $125-137$.

[9] Borgatti S.P., Mehra A., Brass D.J., and Labianca G.(2009). Network Analysis in the Social Sciences. Science, vol. 323, pp. 892-895.

[10] Chen P-Y., and Cheng S-M. (2015). Sequential defense against random and intentional attacks in complex networks. Physical Review E, vol. 91, pp. 022805.

[11] Cinelli M., Ferraro G. and Iovanella A. (2017). Resilience of core-periphery networks in the case of rich-club. Complexity, (ID 6548362):1-12, DOI: 10.1155/2017/6548362. 
[12] Colizza V., Barrat A., Barthlemy M., and Vespignani A. (2006). The role of the airline transportation network in the prediction and predictability of global epidemics. Proceedings of the National Academy of Sciences of the United States of America, vol. 103, pp. 2015-2020.

[13] Colizza V., Pastor-Satorras R. and Vespignani A. (2007). Reaction-diffusion processes and metapopulation models in heterogeneous networks. Nature Physics, vol. 3, no. 4, pp. 276-282.

[14] Csardi G. and Nepusz T., (2006). The igraph software package for complex network research, InterJournal Complex System, vol. 1695, http://igraph.org.

[15] Faloutsos M., Faloutsos P., and Faloutsos C. On power-law relationships of the internet topology.In Proceedings of the ACM SIGCOMM 1999 Conference on Application, Technologies, Architectures, and Protocols for Computer Communication, Cambridge, MA, USA. 0 August- 3 September 1999, vol. 29, pp. 251-262.

[16] Faust K. (2006). Comparing social networks: Size, density and local structure. Metodoloski zvezki, vol. 3, pp. 185-216.

[17] Ferraro G. and Iovanella A. (2018). Clairvoyant targeted attack on complex networks International Journal of Computational Economics and Econometrics, vol. 8, n. 1, pp. 41-61.

[18] Fortune S., Hopcroft J. E. and Wyllie J. (1980). The Directed Subgraph Homeomorphism Problem, Theoretical Computer Science, vol. 10, pp. 111-121.

[19] Fraccascia, L., Giannoccaro, I., and Albino, V. (2018). Resilience of complex systems: state of the art and directions for future research, Complexity, vol. 2018, ID 3421529, DOI: $10.1155 / 2018 / 3421529$.

[20] Francis, R. and Bekera, B. (2014). A metric and framework for resilience analysis of engineered and infrastructure systems, Reliability Engineering and System Safety, vol. 121, pp. 90-103.

[21] Gao, J., Barzel, B. and Barabási, A.-L. (2016). Universal resilience patterns in complex networks, Nature, 530, pp. 307-312. 
[22] Hosseini S., Barker K., Ramirez-Marquez J.E. (2016). A review of definitions and measures of system resilience, Reliability Engineering and System Safety, vol. 145, pp. 47-61.

[23] Karrer, B., Levina, E., and Newman, M.E.J. (2008). Robustness of community structure in networks, Physical Review E, 77, 046119.

[24] Miller-Hooks, E., Zhang, X. and Faturechi, R. (2012). Measuring and maximizing resilience of freight transportation networks, Computers and Operations Research, vol. 39 (7), pp. 1633-1643.

[25] Ramirez-Marquez, J.E., Rocco, C.M., Barker, K. and Moronta, J. (2018). Quantifying the resilience of community structures in networks, Reliability Engineering and System Safety, vol. 169, pp. 466-474.

[26] Rosenkrantz D. J. Goel S., Ravi S. S. and Gangolly J. (2005). Structure-based resilience metrics for service-oriented networks, in: Dal Cin M., Kaniche M., Pataricza A. (eds) Dependable Computing - EDCC 5. EDCC 2005. Lecture Notes in Computer Science, vol 3463. Springer, Berlin, Heidelberg.

[27] R Core Team (2014) R: A Language and Environment for Statistical Computing, $R$ Foundation for Statistical Computing, Vienna, Austria, http://www.R-project.org.

[28] Rual J., Venkatesan K:, Hao T., et al. (2005). Towards a proteome-scale map of the human protein-protein interaction network. Nature, vol. 437, 1173.

[29] Shaw S.-L., Fang Z., Lu S., Tao R. (2014). Impacts of high speed rail on railroad network accessibility in China, Journal of Transport Geography, vol. 40, pp. 112-122.

[30] Smith, P., Hutchison, D., Sterbenz, J.P.G., Schöller, M., Fessi, A., Karaliopoulos, M., Lac, C. and Plattner, B. (2011) Network resilience: a systematic approach, . IEEE Communications Magazine, vol. 49 (7), pp. 88-97.

[31] Sterbenz, J.P.G., Çetinkaya, E.K., Hameed, M.A., Jabbar, A., Qian S. and Rohrer, P. (2011). Evaluation of network resilience, survivability, and disruption tolerance: analysis, topology generation, simulation and experimentation, Telecommunication Systems, vol. 52 (2), pp. 705-736. 
[32] Wang J.W., Gao F., and Ip W.H. (2010). Measurement of resilience and its application to enterprise information systems, Enterprise Information Systems, vol. 4 (2), pp. 215-223, DOI: 10.1080/17517571003754561.

[33] Wang D.W. and Ip W.H. (2009). Evaluation and analysis of logistic network resilience with application to aircraft serving, IEEE Systems Journal, vol. 3 (2), pp. 166-173.

[34] Woods D.D. (2015). Four concepts for resilience and the implications for the future of resilience engineering, Reliability Engineering and System Safety, vol. 141, pp. 5-9.

[35] Zhang, X., Mahadevan, S., Sankararaman, S. and Goebel, K. (2018) Resilience-based network design under uncertainty, Reliability Engineering and System Safety, vol. 169, pp. 364-379. 


\begin{tabular}{|c|c|}
\hline Symbol & Meaning \\
\hline$G$ & The considered graph. \\
\hline$V$ & Set of the nodes of the graph $G$. \\
\hline$E$ & Set of the arcs of the graph $G$. \\
\hline$n$ & Number of the nodes, cardinality of the set $V$. \\
\hline$m$ & Number of the arcs, cardinality of the set $E$. \\
\hline$i, j$ and $i_{0}, i_{1}, \ldots, i_{k}$ & Generic elements of $V$. \\
\hline $\mathbf{A}=\left(a_{i j}\right)_{i, j=1, \ldots, n}$ & Unweighted adjacency matrix of $G$ with related entries $a$ 's. \\
\hline $\mathbf{W}=\left(w_{i j}\right)_{i, j=1, \ldots, n}$ & Weighted adjacency matrix of $G$ with related entries $w$ 's. \\
\hline$N=(V, \mathbf{W})$ & The network given by the graph $G$ and the weighted adjacency matrix $\mathbf{W}$. \\
\hline$p_{i_{0}}^{(k)}$ & $k$-path having $i_{0}$ as starting node. \\
\hline$w\left(p_{i_{0}}^{(k)}\right)$ & weight of the $k$-path $p_{i_{0}}^{(k)}$ \\
\hline $\bar{k}$ & Length of the longest $k$-path of the network. \\
\hline $\mathcal{P}^{(k)}\left(i_{0}\right)$ & Set collecting all the $k$-paths of $N$ with starting node $i_{0}$. \\
\hline $\mathcal{P}^{(k)}$ & Set collecting all the $k$-paths of $N$. \\
\hline$\xi$ & Size of a shock, $\xi \in[0,+\infty)$ \\
\hline$\delta$ & Discount factor, $\delta \in(0,1)$. \\
\hline$t$ & Time. \\
\hline$\xi_{h}$ & Shock propagated at time $t=h$ and in the node $i_{h}$. \\
\hline$\Gamma=\left(\gamma_{1}, \ldots \gamma_{\bar{k}}\right)$ & Vector of the propagation thresholds with components $\gamma$ 's, or PC-vector of the network. \\
\hline$\Gamma^{\star}=\left(\gamma_{1}^{\star}, \ldots \gamma_{\bar{k}}^{\star}\right)$ & Vector of the minimal values of the propagation thresholds, or critical PC-vector of the network. \\
\hline$(\Gamma, \xi)-P C$ & Label of the $k$-paths which satisfy the propagation condition. It depends on $\Gamma$ and $\xi$ \\
\hline $\mathcal{P}_{\Gamma, \xi}^{(k)}$ & Set collecting the $k$-paths which are $(\Gamma, \xi)-P C$ \\
\hline$\Gamma-\xi$-resilience of $N$ & Ability of $N$ to absorb shocks. It depends on $\Gamma$ and $\xi$. \\
\hline$\mu_{(\Gamma, \xi)}(N)$ & Measure of the $\Gamma-\xi$-resilience of $N$. \\
\hline$\Theta=\left(\theta_{1}, \ldots, \theta_{\bar{k}}\right)$ & Vector of the resilience weights associated to the measure $\mu_{(\Gamma, \xi)}$, with the components $\theta$ 's. \\
\hline
\end{tabular}

Table 1: Table of the notation used throughout the paper. 